\title{
EFFECTS OF VISCOUS DISSIPATION AND MAGNETOHYDRODYNAMICS ON REE-EYRINGFLUID FLOW OVER A DEFORMABLE POROUS CHANNEL
}

\author{
A. NEERAJA ${ }^{1 *}$, V. NAGARADHIKA ${ }^{2}$, R. L. V. RENUKA DEVI ${ }^{3}$ \& P. S. S. R. SUJATHA ${ }^{4}$ \\ ${ }^{1,4}$ Department of Mathematics, Aditya College of Engineering, Surampalem, Andhra Pradesh, India \\ ${ }^{2}$ Department of Mathematics, GITAM Deemed to be University, Bengaluru, Karnataka, India \\ ${ }^{3}$ Department of Mathematics, Sri Venkateswara University, Tirupati, Andhra Pradesh, India
}

\begin{abstract}
In this analysis magneto hydrodynamics (MHD) flow of Ree-Eyring fluid flow over a deformable porous channel is studied. The governing partial differential equations are transformed to ordinary differential equations by using the nondimensional quantities. The governing non-linear differential equations are solved by using Runge-Kutta fourth order method along with shooting technique. The impacts of magnetic parameter, Ree-Eyring parameter, viscous drag, angle of inclination, lower and upper plate moving velocities, heat source parameter, Brinkmann number and volume fraction parameter on the fluid velocity, the solid displacement and the temperature distribution are shown with the help of graphs. The mass flow rate is found in numerically and shown it tabular form. The fluid velocity enhances with increasing on-Newtonian parameter.

KEYWORDS: Magneto Hydrodynamics Flow; Ree-Eyring Fluid; Shooting Technique \& Mass Flow Rate.
\end{abstract}

Received: Jun 06, 2020; Accepted: Jun 26, 2020; Published: Jul 29, 2020; Paper Id.: IJMPERDJUN2020521

\section{NOMENCLATURE}

\begin{tabular}{|l|l}
\hline$\mu_{a}$ is the apparent viscosity of the fluid in the & $\begin{array}{l}U \text { is the average velocity } \\
\text { porous material }\end{array}$ \\
$K$ is the drag coefficient & $\beta$ is the permeability parameter \\
$\mu$ is the Lame constant & $R$ is the volume fraction of the fluid \\
$\theta$ is the angle of inclination & $F r$ is the Froude number \\
$K_{0}$ is the thermal conductivity & $\theta$ is the angle of inclination \\
$v$ is the fluid velocity & $\delta$ is the viscous drag coefficient, \\
$Q_{0}$ is the constant heat source & $M$ is the magnetic parameter \\
$\phi$ is the volume fraction of the fluid & $P$ is the pressure gradient \\
$u$ is the solid displacement & $B r$ is the Brinkman number \\
$\rho$ is the fluid density
\end{tabular}




\begin{tabular}{|l|l|}
\hline$g$ is the gravity & $\beta_{1}$ is the constant heat source parameter \\
$\frac{\partial p}{\partial x}$ is the pressure gradient & $U_{1}$ and $U_{2}$ are the lower and upper plate \\
$\sigma$ is the electrical conductivity & moving velocities. \\
$B_{0}$ is the strength of the magnetic field & $\bar{B}, \bar{C}$ are the material constants of Ree-Eyring \\
& fluid model \\
\hline
\end{tabular}

\section{INTRODUCTION}

Recently, the researchers study of Newtonian and non-Newtonian fluids has gained much intension and its widespread applications in Engineering and Industrial areas. Newtonian fluid noting but the fluids which obey the Newton's law of viscosity otherwise it is called non-Newtonian fluid. Examples of Newtonian fluids are water, gas, etc. and physiological liquids, pharmaceutical products, blood etc are the examples of non-Newtonian fluid. Ree-Eyring fluid model is one of the non-Newtonian fluids. Taha [1] studied variational approach for the flow of Ree-Eyring and Casson fluids in pipes. Ramesh et al. [2] developed effects of thermal radiation and MHD on Ree-Eyring fluid flows through porous medium with slip boundary conditions. Ijazet al. [3] reported peristaltic propulsion of particulate non-Newtonian Ree-Eyring fluid in a through duct through constant magnetic field. Heat transfer analysis on peristaltic transport of Ree-Eyring fluid in rotating frame was examined by Hayat et al. [4]. Bhatti et al. [5] founded combine effect of MHD and partial slip on peristaltic blood flow of Ree-Eyring fluid with wall properties. Ijazet al. [6] developed heat transfer analysis in MHD flow of solid particles in nonNewtonian Ree-Eyring fluid due to peristaltic wave in a channel. Entropy generation in radiative flow of Ree-Eyring fluid due to rotating disks was described by Ijaz Khan et al. [7].Hayat et al. [8] studied theoretical investigation of ReeEyringnanofluid flow with entropy optimization and Arrhenius activation energy between two rotating disks. Krishna Murthy et al. $[9,10]$ developed hydormagnetic flow of Casson fluid through a vertical deformable porous stratum with viscous dissipation and chemical reaction and effects of heat and mass transfer flow of a Jeffrey fluid through a vertical deformable porous stratum. Sreenadhet al. [11] analyzed MHD Couette flow of a Jeffrey fluid over a deformable porous layer. Sudhakaraet al. [12] discussed effect of heat transfer on free surface flow of a Jeffrey fluid over a deformable permeable bed.MHD Couette flow of Jeffrey fluid in a porous channel with heat source and chemical reaction was developed by Krishna Murthy [13]. Sreenadhet al. [14] discussed MHD free surface flow of a Jeffrey fluid over a deformable porous layer. Krishna Murthy [15,16] studied MHD Poiseuille flow of Jeffrey fluid over a deformable porous layer and Couette flow over a deformable permeable bed. Barry et al. [17] investigated on Fluid flow over a thin deformable porous layer. DariuszGawinet al. [18] discussed coupled heat, water and gas flow in deformable porous media. Entropy generation analysis for MHD flow through a vertical deformable porous layer was developed by Sreenadhet al. [19].An entropy generation on viscous fluid in the inclined deformable porous medium was reported by Gopi Krishnaet al. [20]. Nieldet al. [21] described effects of viscous dissipation and flow work on forced convection in a channel filled by a saturated porous medium.

Present study deals with hydromagnetic flow of Ree-Eyring fluid flow over a deformable porous channel. The governing equations of the fluid velocity, the solid displacement and the concentrations are solved numerically with shooting technique. The effects of governing parameters on the fluid velocity, the solid displacement and the concentrations are shown graphically. The mass flow rate is shown numerically in tabulated form. The present results have been good agreement with 
existing studies under some special cases.

\section{MATHEMATICAL FORMULATION OF THE PROBLEM}

Consider the steady hydromagnetic flow of Power-Eyring fluid over a deformable porous layer with solid walls at $y=-h$ and $y=h$ as shown in Figure 1. The lower and upper plates are moving with distinct velocities $U_{1}$ and $U_{2}$ respectively. The temperature are maintained at the plates are $T_{w}$ and $T_{0}$ respectively. The fluid flow along the $x-$ axis and the $y-$ axis is perpendicular to it. The uniform magnetic field of strength $B_{0}$ is applied perpendicular to the plates of channel. A pressure gradient $\frac{\partial p}{\partial x}$ is applied producing an axially directed flow in the channel.

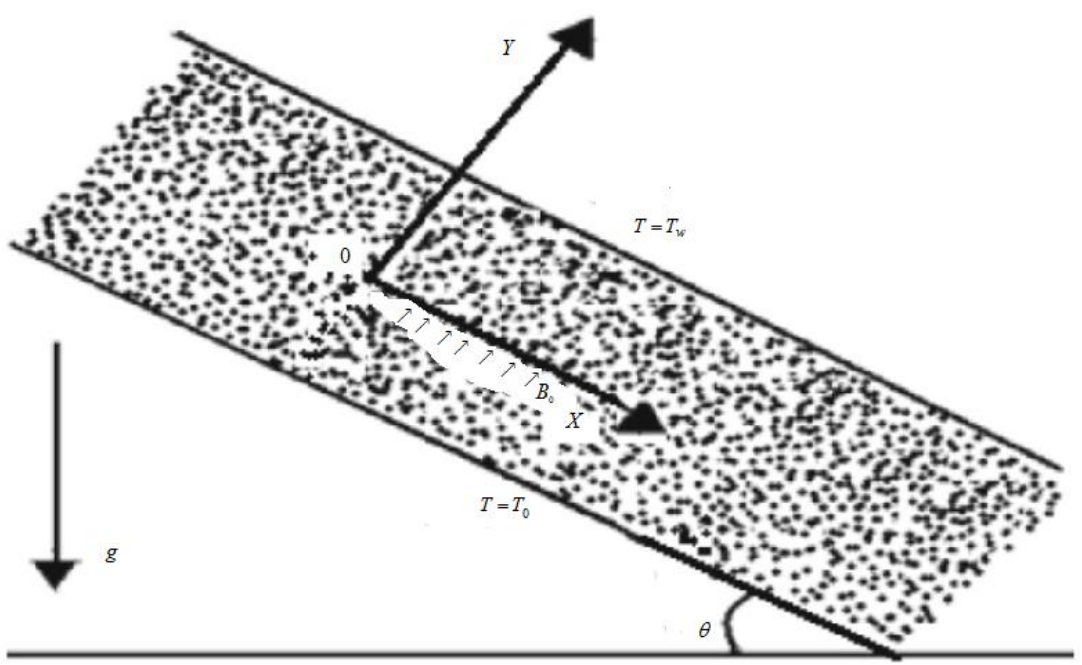

Figure 1: Geometry Flow of the Problem.

The governing equations of the fluid velocity, the solid displacement and the temperature are as follows:

$$
\begin{gathered}
2 \mu_{a}\left(1+\frac{1}{\mu_{a} \bar{B} \bar{C}}\right) \frac{\partial^{2} v}{\partial y^{2}}-\phi \frac{\partial p}{\partial x}-\sigma B_{0}^{2} v+\rho g \sin \theta-K v=0 \\
\mu \frac{\partial^{2} u}{\partial y^{2}}-(1-\phi) \frac{\partial p}{\partial x}+\rho g \sin \theta+K v=0 \\
K_{0} \frac{\partial^{2} T}{\partial y^{2}}+2 \mu_{a}\left(1+\frac{1}{\mu_{a} \bar{B} \bar{C}}\right)\left(\frac{\partial v}{\partial y}\right)^{2}+Q_{0}=0
\end{gathered}
$$

The boundary conditions are:

$$
\left.\begin{array}{l}
v=U_{1}, u=0, T=T_{0} \text { at } y=-h \\
v=U_{2}, u=0, T=T_{1} \text { at } y=h
\end{array}\right\}
$$

Now introducing the non-dimensional quantities are as follows: 


$$
\left.\begin{array}{l}
F r=\frac{U}{g h}, U_{1}^{*}=\frac{U_{1}}{U}, U_{2}^{*}=\frac{U_{2}}{U}, y^{*}=\frac{y}{h}, v^{*}=\frac{v}{U}, x^{*}=\frac{x}{h}, \theta^{*}=\frac{T-T_{0}}{T_{1}-T_{0}}, \\
p^{*}=\frac{h p}{2 \mu_{a} U}, u^{*}=\frac{u \mu}{2 \mu_{a} U}, \frac{d p}{d x}=P, \delta=\frac{K h^{2}}{2 \mu_{a}}, \operatorname{Re}=\frac{\rho U h}{2 \mu_{a}}, \beta_{1}=Q_{0}\left(\frac{h^{2}}{K_{0}\left(T_{w}-T_{0}\right)}\right), \\
\operatorname{Br}=\frac{2 \mu_{a} U^{2}}{K_{0}\left(T_{w}-T_{0}\right)}, M^{2}=\frac{\sigma B_{0}^{2} h^{2}}{\mu}, \beta=\frac{1}{\mu_{a} \bar{B} \bar{C}}
\end{array}\right\}
$$

Using equation (5) in equations (1)-(4) and after neglecting the asterisks (*) takes the following equations are:

$$
\begin{aligned}
& (1+\beta) \frac{d^{2} v}{d y^{2}}-\phi P+\frac{\operatorname{Re}}{\operatorname{Fr}} \sin \theta-\left(\delta+M^{2}\right) v=0 \\
& \frac{d^{2} u}{d y^{2}}-(1-\phi) P+\frac{\operatorname{Re}}{\operatorname{Fr}} \sin \theta+\delta v=0 \\
& \frac{d^{2} \theta}{d y^{2}}+B r(1+\beta)\left(\frac{d v}{d y}\right)^{2}+\beta_{1}=0
\end{aligned}
$$

The boundary conditions are

$$
\left.\begin{array}{l}
v=U_{1}, u=0, \theta=0 \text { at } y=-1 \\
v=U_{2}, u=0, \theta=1 \text { at } y=1
\end{array}\right\}
$$

\section{Mass Flow Rate}

The non dimensional mass flow rate $M_{1}$ per unit width of the channel as follows:

$$
M_{1}=\int_{0}^{1} v d y
$$

We are interest to finding the Shear stress and the Nusselt number as follows:

$$
\tau=(1+\beta) v^{\prime}(1), N u=-\theta^{\prime}(1)
$$

\section{RESULTS AND DISCUSSIONS}

In this paper we investigated the impacts of viscous dissipation and magneto hydrodynamics flow of Ree-Eyring fluid over a deformable porous channel. The governing non-linear ordinary differential equations are solved by using Runge-Kutta fourth order method along with shooting technique. The influence of governing parameters on the fluid velocity, the solid displacement and the temperature are shown in graphically. The mass flow rate is calculated numerically and shown in tabular form. The present results have been good agreement when compared with existing results under some special cases.

The variation of Ree-Eyring fluid parameter $\beta$ on the fluid velocity $v(y)$ and the solid displacement $u(y)$ is shown in Figures 2 and 3. We noticed that both the fluid velocity and the solid displacement are enhancing with increasing 
Ree-Eyring fluid parameter. The impact of magnetic parameter $M$ on the fluid velocity $v(y)$ and the solid displacement $u(y)$ is represented in Figures 4 and 5. We reveal that both the fluid velocity and the solid displacement are decays for higher values of magnetic parameter. This causes an increase in magnetic parameter in an increase in the Lorentz force. The behavior of Lorentz force is resisting the flow, when we increase the magnetic parameter, Lorentz forces increases. figures 6-9 represents the effects of lower plate $U_{1}$ and upper plate $U_{2}$ moving velocities on the fluid velocity $v(y)$ and the solid displacement $u(y)$. We have seen that both the fluid velocity and solid displacement are increases with increasing moving velocities. The variation of the volume fraction coefficient $\phi$ on the fluid velocity $v(y)$ and the solid displacement $u(y)$ is shown in figures 10 and 11 . We noticed that the fluid velocity greater with increasing volume fraction coefficient and the opposite behavior observed in the solid displacement. From figures 12 and 13 elucidated the influence of viscous drag $\delta$ on the fluid velocity $v(y)$ and the solid displacement $u(y)$ are executed. We reveal that the fluid velocity decays for higher values of viscous drag and the opposite nature we have seen in the solid displacement. These causes the drag forces are acting in opposite direction to the relative motion of the object which always opposes the motion. Therefore the fluid velocity decays when the drag enhances. The impact of the angle of inclination $\theta$ on the fluid velocity $v(y)$ and the solid displacement $u(y)$ is displayed in figures 14 and 15 . We examined that both the fluid velocity and the solid displacement are enhance with enhancing the angle of inclination. The variation of Brinkman number $\mathrm{Br}$ on the temperature distribution $\theta(y)$ is sketched in figure 16. It reveals that the temperature enhances with increasing Brinkman number. This causes for higher values of Brinkman number implies a lower contribution in thermal conduction generated via viscous dissipation and greater elevation in temperature. The influence of heat source/sink parameter $\beta_{1}$ on the temperature distribution $\theta(y)$ is displayed in figure 17. We noticed that the temperature increases with increasing heat source/sink parameter.

The numerical values of the mass flow rate $M_{1}$ with deformable porous medium and undeformable porous medium are shown in Table 1. We noticed that the volume flow rate $M_{1}$ with deformable porous medium reduces for higher values of drag coefficient $\delta$ for fixed value of $\phi=0.6$. The same nature is noticed that in the case of mass flow rate $M_{1}$ with undeformable porous medium Nieldet al. [21] for higher values of Darcy number $D a$. Present results have been good agreement with existing studies Nieldet al. [21] when $M=0, \beta=0, \theta=0$ and drag parameter $\delta$ in deformable porous medium is equal to the Darcy number $D a$ porous medium in a channel for the case of $\phi=1$.

\section{CONCLUSIONS}

In this paper magneto hydrodynamics flow of a Ree-Eyring fluid over a deformable porous channel is analyzed. The nonlinear coupled differential equations are solved using shooting technique. The effects of governing parameters on the fluid velocity, the solid displacement and the temperature are shown in graphically. The mass flow rate is calculated numerically and displayed in tabular form. The objectives of the present paper are as follows:

- The fluid velocity and the solid displacement are enhancing with the influence of Ree-Eyring fluid parameter, angle 
of inclination, lower and upper plate moving velocities.

- The temperature enhances with an impact of Brinkmann number and constant heat source parameter.

- The fluid velocity and the solid displacement are decreases with the influence of magnetic parameter.

- The variation of viscous drag the velocity reduces and the opposite nature observed in sold displacement. The fluid velocity and the solid displacement are having opposite behavior with the influence of volume fraction coefficient.

- The present results have been good agreement when compared with Gopi Krishna et al.[20] and Nieldet al. [21] under some special cases.

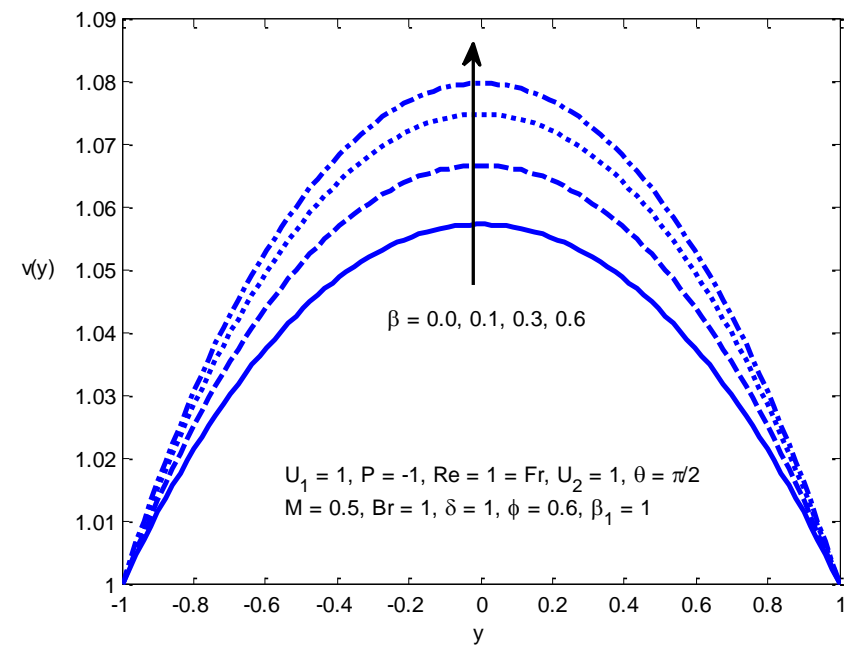

Figure 2: The Impact of $\beta$ on the Fluid Velocity $v(y)$

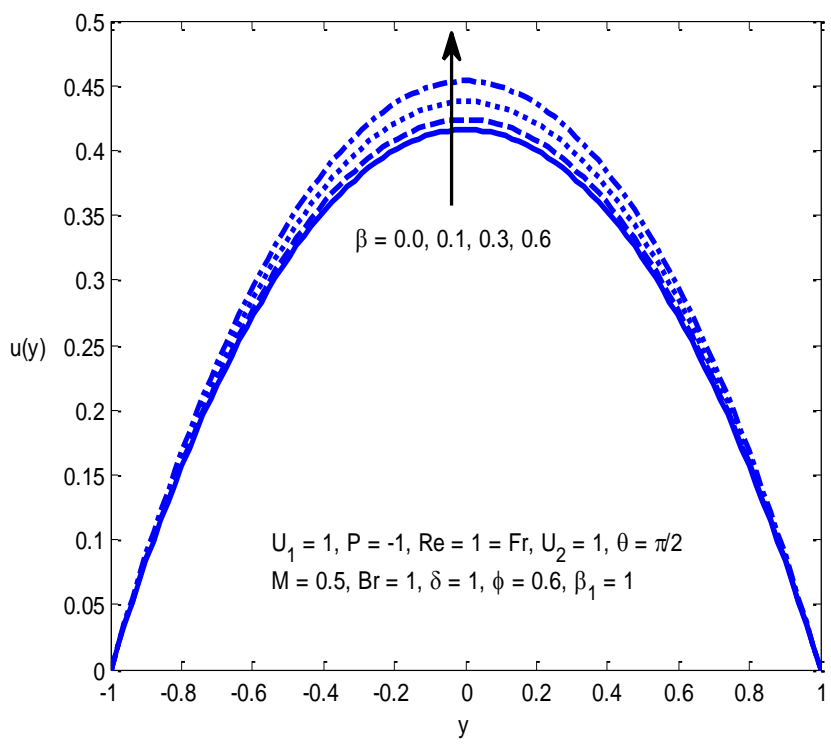

Figure 3. The Impact of $\beta$ on the Solid Displacement $u(y)$. 


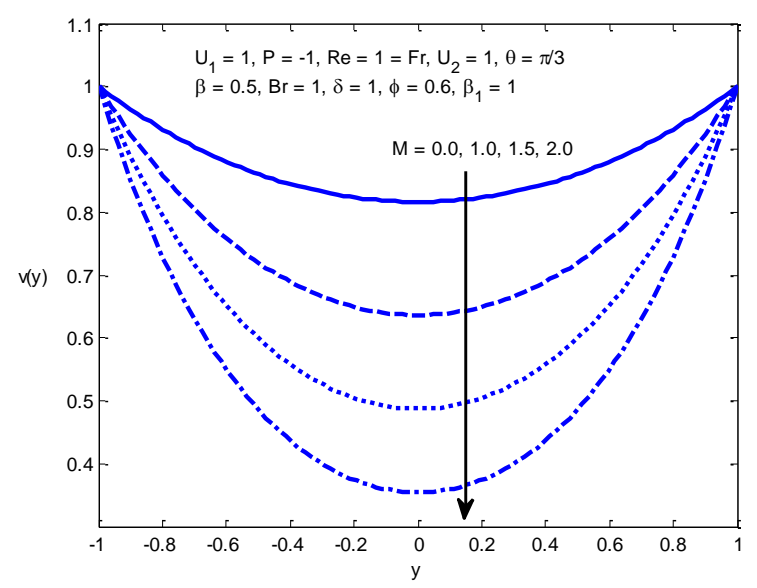

Figure 4: The Impact of $M$ on the Fluid Velocity $v(y)$.

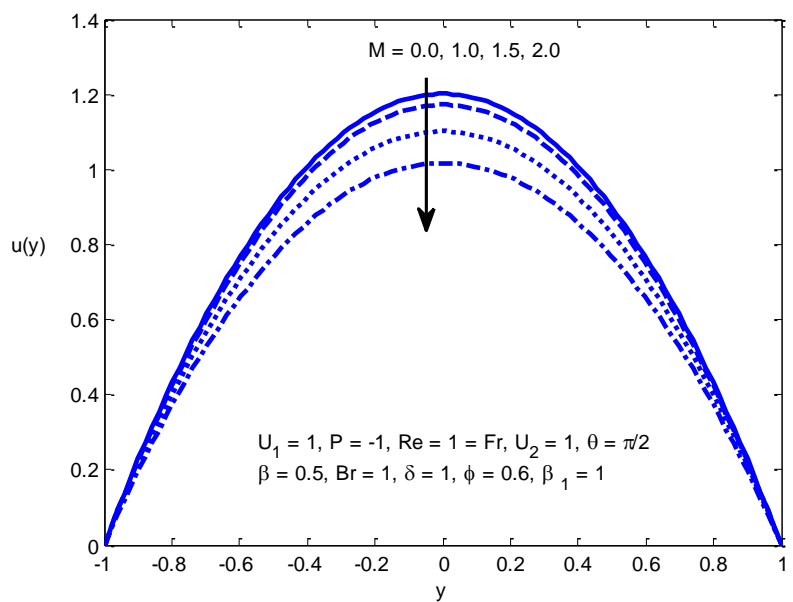

Figure 5: The Impact of $M$ on the Solid Displacement $u(y)$.

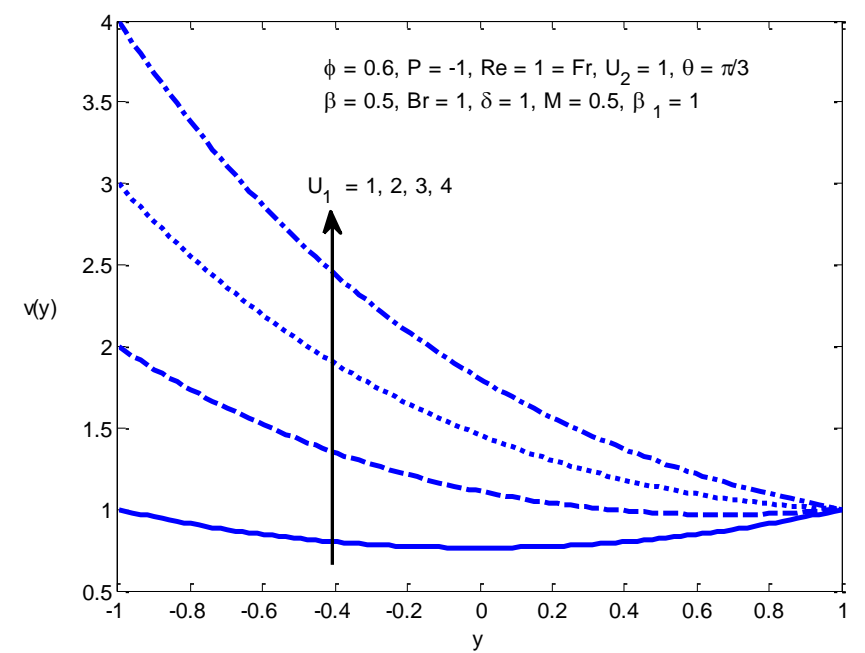

Figure 6: The Impact of $U_{1}$ on the Fluid Velocity $v(y)$. 


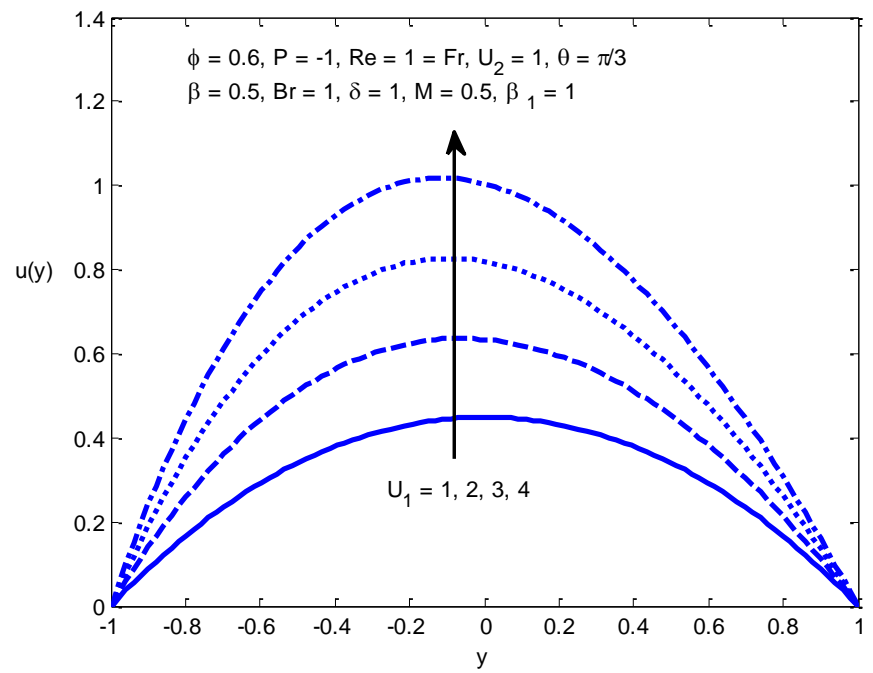

Figure 7: The Impact of $U_{1}$ on the Solid Displacement $u(y)$.

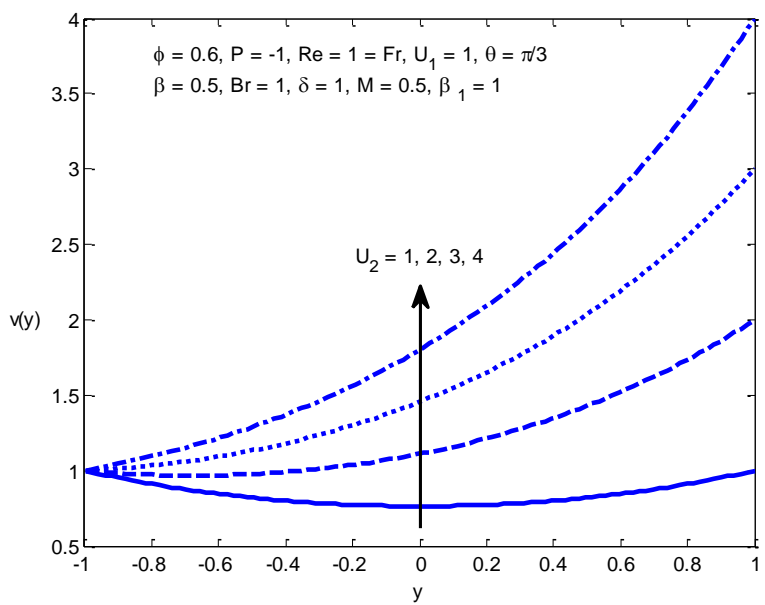

Figure 8: The Impact of $U_{2}$ on the Fluid Velocity $v(y)$.

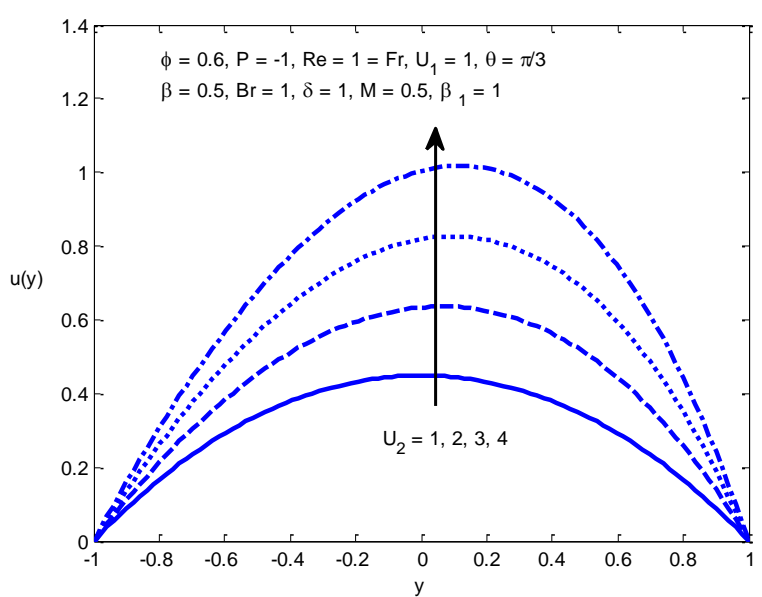

Figure 9: The Impact of $U_{2}$ on the Solid Displacement $u(y)$. 


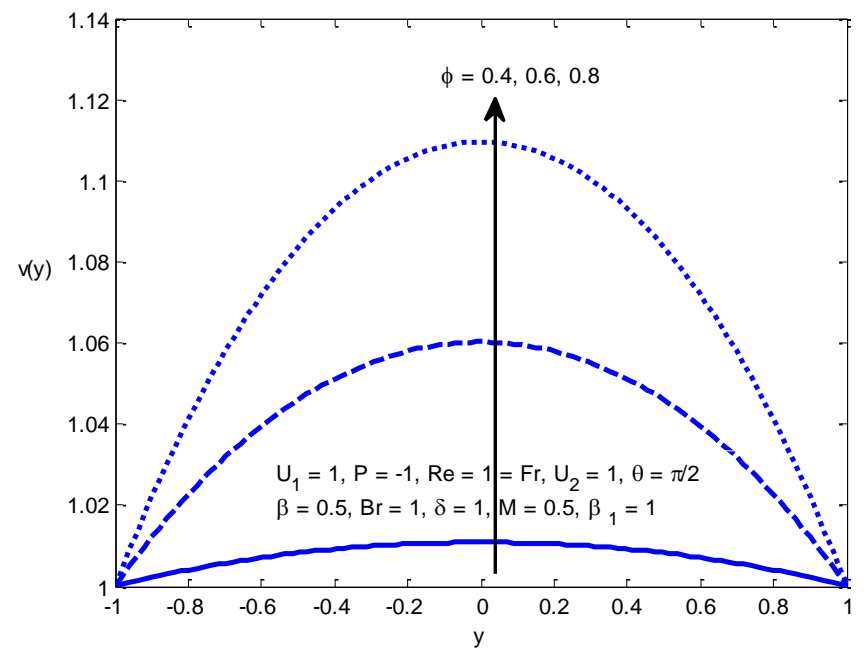

Figure 10: The Impact of $\phi$ on the Fluid Velocity $v(y)$

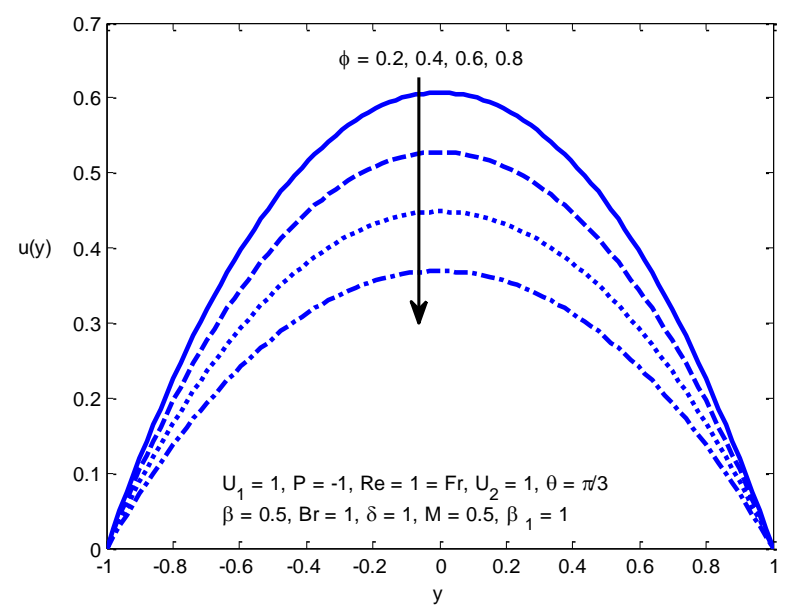

Figure 11: The Impact of $\phi$ on the Solid Displacement $u(y)$.

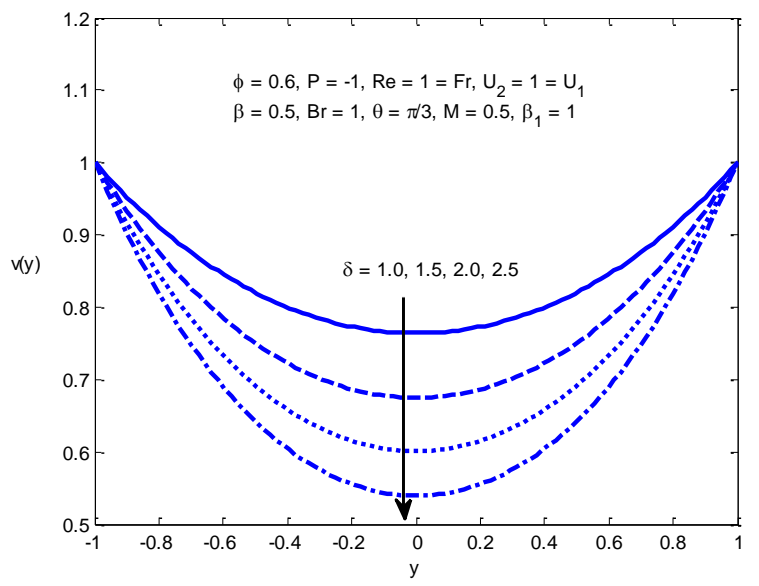

Figure 12: The Impact of $\delta$ on the Fluid Velocity $v(y)$ 


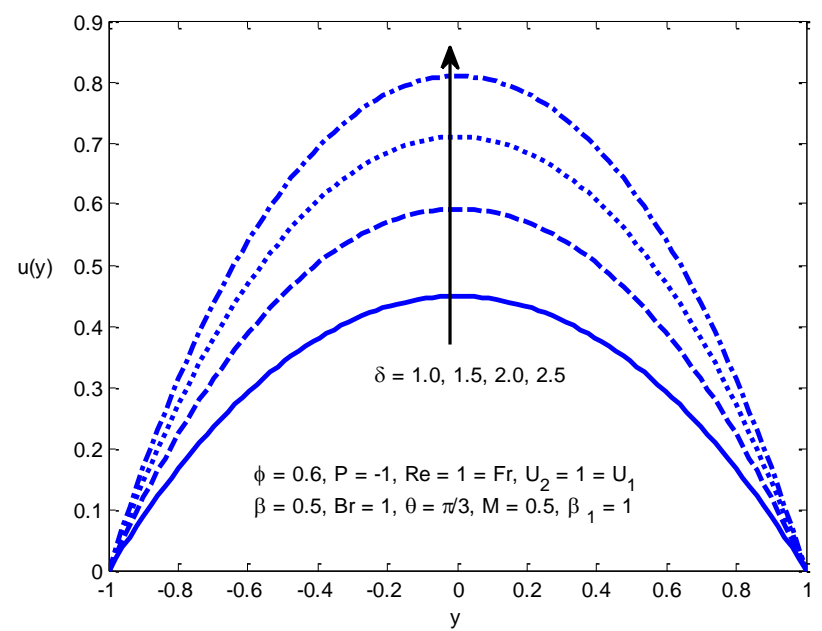

Figure 13: The Impact of $\delta$ on the Solid Displacement $u(y)$.

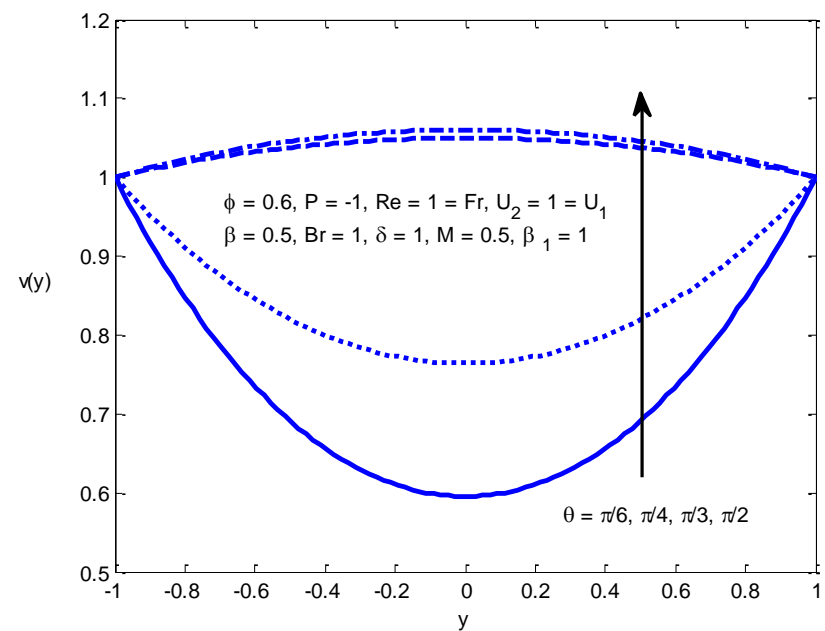

Figure 14: The Impact of $\theta$ on the fluid Velocity $v(y)$.

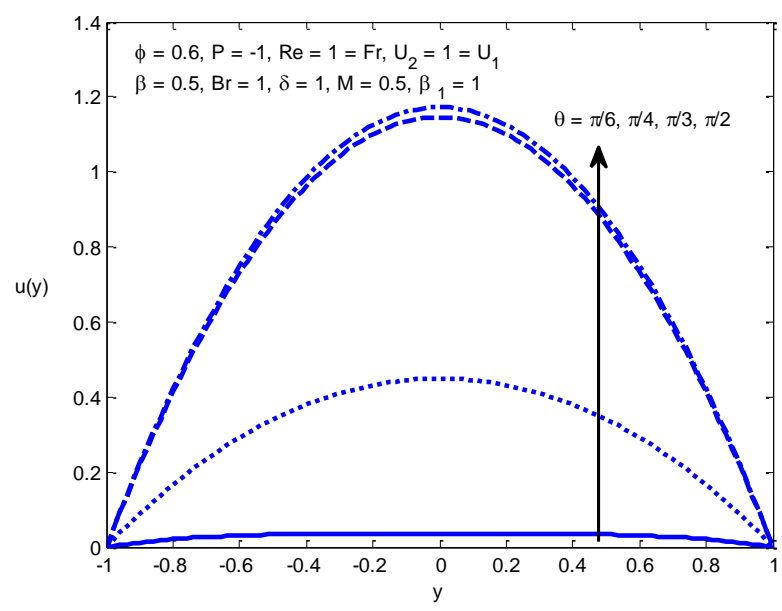

Figure 15: The Impact of $\theta$ on the Solid Displacement $u(y)$. 


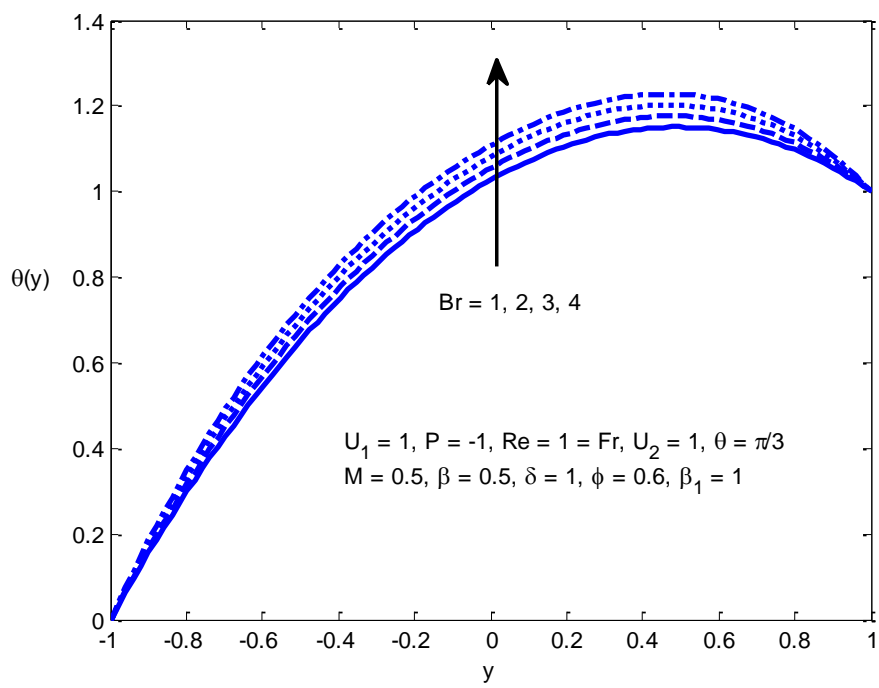

Figure 16: The Impact of $B r$ on the Temperature Distribution $\theta(y)$.

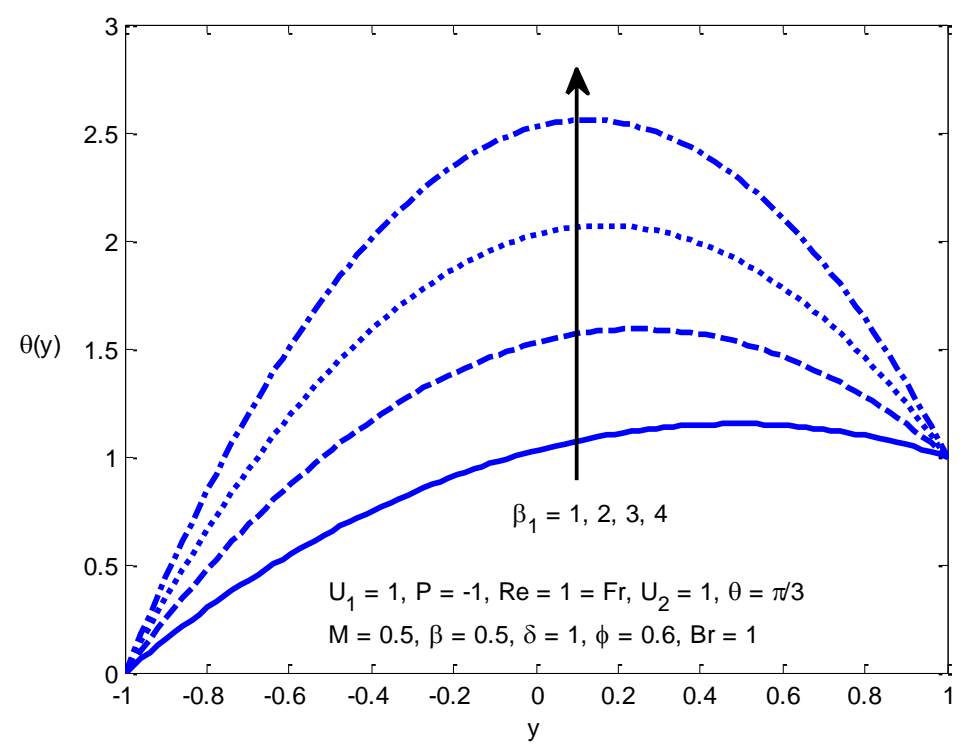

Figure 17: The Impact of $\beta_{1}$ on the Temperature Distribution $\theta(y)$.

Table 1: Numerical Values of Mass Flow Rate $M_{1}$ with Distinct Values of $D a$ and $\delta$

\begin{tabular}{|c|c|c|c|c|c|c|c|}
\hline \multicolumn{2}{|c|}{$\begin{array}{c}\text { Nieldet al. [20] } \\
\text { (with Porous Medium) }\end{array}$} & \multicolumn{2}{|c|}{$\begin{array}{c}\text { Gopi Krishna } \text { et al.[21] } \theta=\mathbf{0} \\
\text { (with Deformable Porous Medium) }\end{array}$} & \multicolumn{2}{c|}{$\begin{array}{c}\text { Present Work } M=0, \beta=0, \theta=0 \\
\text { (with Deformable Porous Medium) }\end{array}$} \\
\hline \multirow{2}{*}{$\boldsymbol{D} a$} & $M_{1}$ & $\delta$ & $\begin{array}{c}M_{1} \\
\text { (when } \phi=\mathbf{0 . 6})\end{array}$ & $\begin{array}{c}M_{1} \\
\text { (when } \phi=1)\end{array}$ & $\delta$ & $\begin{array}{c}M_{1} \\
\text { (when } \phi=0.6)\end{array}$ & $\begin{array}{c}M_{1} \\
\text { (when } \phi=1)\end{array}$ \\
\hline 1 & 0.4768 & 1 & 0.2861 & 0.4768 & 1 & 0.28612 & 0.47683 \\
1.5 & 0.4177 & 1.5 & 0.2506 & 0.4177 & 1.5 & 0.25064 & 0.41775 \\
2 & 0.3718 & 2 & 0.2231 & 0.3718 & 2 & 0.22316 & 0.37187 \\
2.5 & 0.3351 & 2.5 & 0.2011 & 0.3351 & 2.5 & 0.20118 & 0.33519 \\
\hline
\end{tabular}




\section{REFERENCES}

1. S. Taha, Variational approach for the flow of Ree-Eyring and Casson fluids in pipes, Int. J. Mod. Sim. Sci. Comp., 7 (2016) 1650007.

2. K. Ramesh, Sartaj Ahmad Eytoo, Effects of thermal radiation and MHD on Ree-Eyring fluid flows through porous medium with slip boundary conditions, Mul. Mod. Mat. Strucs., 15 (2019) 492-507.

3. N. Ijaz, A. Zeeshan, M.M. Bhatti, Peristaltic propulsion of particulate non-Newtonian Ree-Eyring fluid in a through duct through constant magnetic field, Alexandria. Eng. J., 57 (2018) 1055-1060.

4. T. Hayat, H. Zahir, A. Alsedi, B. Ahmad, Heat transfer analysis on peristaltic transport of Ree-Eyring fluid in rotating frame, Chi. J. Phys., 55 (2017) 1894-1907.

5. M.M. Bhatti, M.A. Abbas, M.M. Rashidi, Combine effect of MHD and partial slip on peristaltic blood flow of Ree-Eyring fluid with wall properties, Eng. Sci. Tech. Int. J., 19 (2016) 1497-1502.

6. Job, Victor M., and S. Rao Gunakala. "Unsteady MHD Free Convection Couette Flow between Two Vertical Permeable Plates in the Presence of Thermal Radiation Using Galerkin's Finite Element Method." International Journal of Mechanical Engineering 2.5 (2013): 99-110.

7. N. Ijaz, M.M. Bhatti, Ahmed Zeeshan, heat transfer analysis in MHD flow of solid particles in non-Newtonian Ree-Eyring fluid due to peristaltic wave in a channel, Ther. Sci., 23 (2019) 1017-1026.

8. M. Ijaz Khan, S.A. Khan, T. Hayat, M.F. Javed, A. Alsaedi, Entropy generation in radiative flow of Ree-Eyring fluid due to rotating disks, Int. J. Num. Meth. Heat \& Fluid Flow, 29 (2019) 2057-2079.

9. T. Hayat, Sohail A Khan, M. Ijaz Khan, A. Alsaedi, Theoretical investigation of Ree-Eyring nanofluid flow with entropy optimization and Arrhenius activation energy between two rotating disks, Comp. Meth. Prog. Biomedicine, 177 (2019) 57-68.

10. Senapati, N., R. K. Dhal, and K. Ray. "Effect of chemical reaction on MHD free convection on Kuvshinshki fluid through porous medium in presence of heat radiation with constant heat and mass flux across moving plate." International Journal of Applied Mathematics \& Statistical Sciences 2.4 (2013): 45-52.

11. M. Krishna Murthy, N. Mahesh Babu, R.L.V. Renukadevi, M.Eswara Rao, Hydormagnetic flow of Casson fluid through a vertical deformable porous stratum with viscous dissipation and chemical reaction, Int. J. Mech. Eng. Tech., 9(2018) 846-854.

12. M. Krishna Murthy, N. Mahesh Babu, R.L.V. Renukadevi, M. Eswara Rao, Effects of heat and mass transfer flow of a Jeffrey fluid through a vertical deformable porous stratum, Int. J. Mech. Eng. Tech., 9(2018) 228-235.

13. S. Sreenadh, K.V. Prasad, H. Vaidya, E. Sudhakara, G. Gopi Krishna, M.Krishna Murthy, MHD Couette flow of a Jeffrey fluid over a deformable porous layer, Int. J. Appl. Comp. Math, 3(2017) 2125-2138.

14. Srihari, K., and Chirra Kesava Reddy. "Effects of Soret and Magnetic Field on Unsteady Flow of a Radiating and Chemical Reacting Fluid: A Finite Difference Approach." International Journal of Mechanical Engineering 3.3 (2014).

15. E. Sudhakara, S. Sreenadh, M. Krishna Murthy, M. Eswara Rao, Effect of heat transfer on free surface flow of a Jeffrey fluid over a deformable permeable bed, Middle. East. J. Sci. Res., 24 (2016)603-612.

16. M. Krishna Murthy, MHD Couette flow of Jeffrey fluid in a porous channel with heat source and chemical reaction, Middle. East. J. Sci. Res., 24(2016) 585-592.

17. S. Sreenadh, M. Krishna Murthy, E. Sudhakara, G.Gopi Krishna,D. Venkateswarlu Naidu, MHD free surface flow of a Jeffrey fluid over a deformable porous layer, Global. J. Pure. Appl. Math., 11 (2015)3889-3903. 
18. M. Krishna Murthy, MHD Poiseuille flow of Jeffrey fluid over a deformable porous layer, Chem. Process Eng. Res., 38(2015) 8-24.

19. Rao, B. Madhusudhana, G. Viswanatha Reddy, and M. C. Raju. "Unsteady MHD mixed convection of a viscous double diffusive fluid over a vertical plate in porous medium with chemical reaction, Thermal radiation and joule heating. "International Journal of Applied Mathematics \& Statistical Sciences (IJAMSS) 2.5 (2013): 93-116.

20. M. Krishna Murthy, E.Sudhakara, G. Gopi Krishna, S. Sreenadh, Couette flow over a deformable permeable bed, Int. J. Innovative. Res. Sci. Eng., (2014)1-9.

21. S.I.Barry, K.H. Parkerf, G.K. Aldis, Fluid flow over a thin deformable porous layer, ZAMP., 45 (1991)633-648

22. DariuszGawin, Paolo Baggio, Bernhard A. Schrefler, Coupled heat, water and gas flow in deformable porous media, Int. J. Num. Fluids., 20 (1995)969-987.

23. Sreenadh, S., Gopi Krishna, G., Srinivas, A.N.S., Sudhakara, Entropy generation analysis for MHD flow through a vertical deformable porous layer, J.Por.Media, 21(2018) 523-538.

24. G. Gopi Krishna, S. Sreenadh, A.N.S. Srinivas, An entropy generation on viscous fluid in the inclined deformable porous medium, Differ.Equ. Dyn. Syst., (2018)1-24.

25. D A Nield, A V Kuznetsov, Ming Xiong, Effects of viscous dissipation and flow work on forced convection in a channel filled by a saturated porous medium, Trans porous media, 56 (2004) 351-367. 
